\title{
USE OF GAMING AND COMPUTER VISION TO DRIVE STUDENT MOTIVATION IN REMOTE LEARNING LAB ACTIVITIES
}

\author{
Franck Luthon ${ }^{1}$, Benoît Larroque ${ }^{1}$, Fawzi Khattar ${ }^{1,2}$, Fadi Dornaika ${ }^{2}$ \\ ${ }^{1}$ Univ. Pau \& Pays Adour UPPA, Anglet (FRANCE) \\ ${ }^{2}$ Univ. Pais Vasco UPV-EHU, San Sebastian (SPAIN)
}

\begin{abstract}
E-learning is now widely used at university level to replace traditional lectures and exercises by digital document reading, quizzes, tutorials, videos, demos and simulations. However the most challenging part is to replace hands-on labs by virtual or remote labs. The difficulty is not only tied to technology (real-time synchronism, scheduling 24/24-7/7, security, mobility, interaction) but also to psychology and cognition aspects. Indeed in distance learning, there is no physical presence of a teacher or tutor to supervise students and drive their motivation, so that the student-teaching process becomes an open loop. Therefore there is a need to find a substitute for the teacher, thanks to technology help, in order to make it a closed-loop process, that is regulated even if there is no teacher in the loop. One needs to be able to evaluate the student mood (concentrated, bored, amused, perplexed...) and to boost the motivation in learning with appropriate tools (immersion, gaming, feedback).
\end{abstract}

Here we address the case of a remote lab in electronics for undergraduate students and we evaluate both the motivation and satisfaction of students experiencing this distant practical activity.

We have developed a platform called LaboREM (for Remote Laboratory) that is in use since 2011. It consists of a hardware setup accessible through the web (server with instruments and Device Under Test DUT). In addition to the electronic setup, a robotic arm and a mobile camera are used to mimic the hand and the eye of the student that might see the lab and act on it by choosing components to build the DUT he wants to characterize. The lab architecture was presented at ICERI'15 and the use of computer vision to enhance the lab was proposed in Edulearn16. A teaser illustrating the concept is available in French: www.youtube.com/watch?v=98b1j5HIqn0

On the server side, two cameras are used: a wide-angle Pan-Tilt-Zoom camera that is static, and a micro-camera onboard a quadcopter-minidrone that can fly in the lab on student's request, to see precisely a specific part of the equipment (e.g. front panel of an oscilloscope). This increases immersion of the student in the remote lab and makes him the actor of the activity (instead of remaining passive).

On the client side, to infer the state of mind of the distant student, a webcam captures the video of his face. Image processing and pattern recognition are used to analyse face expressions and to detect motion (head, eyebrows, mouth etc). Depending on the captured information, various actions may be proposed (e.g. make a pause, be quite, check something) and signals may be sent to alert the student. This allows to control the student behavior and compare it with a normal state.

As regards the pedagogical strategy, a game-like approach is implemented: it is based both on the use of a Top 10 (best anserws to questions during the measurement real-time activity) and on the use of a track-game to find the right solution to an unknown circuit. This is inspired by e-games scenario (levels, lives, points, timer).

Before and after the learning activity, the students answer questionnaires about satisfaction, motivation and psychological profile. From those questionnaires and from the scores obtained in the lab, we analyse the impact of game, robot, drone and camera video processing on the teaching process. We show that the behavior of students is highly affected by these tools, in a positive way as regards motivation.

Keywords: E-learning, electronics, face analysis, game-based learning, labwork, mobile camera, pattern recognition, quadcopter drone, remote lab, student motivation.

\section{INTRODUCTION}

Remote labs constitute an interesting and novel way of doing labs [1,2]. Anywhere and at anytime, the student can access the lab equipment to do his labwork via Internet. This novel way of distance 
learning can be used to increase the motivation for learning of nowadays students, especially if it is coupled with the use of new technologies like quadcopters.

However, apart from technological aspects, one must address the specific point of remote labs (R$L A B$ ), namely the absence of a teacher during the activity, which might lead to a learning process that is uncontrolled, since it becomes an open-loop process [3]. Fig. 1 illustrates the classical situation of hands-on labs with the presence of a human tutor: the teacher both acts as a controller to give advices, hints and proper help or commands to the student, and also as an observer to measure the quality of the student output so that he can estimate the error and regulate the process in a closedloop that hopefully converges towards the desired solution or motivation state of the student.

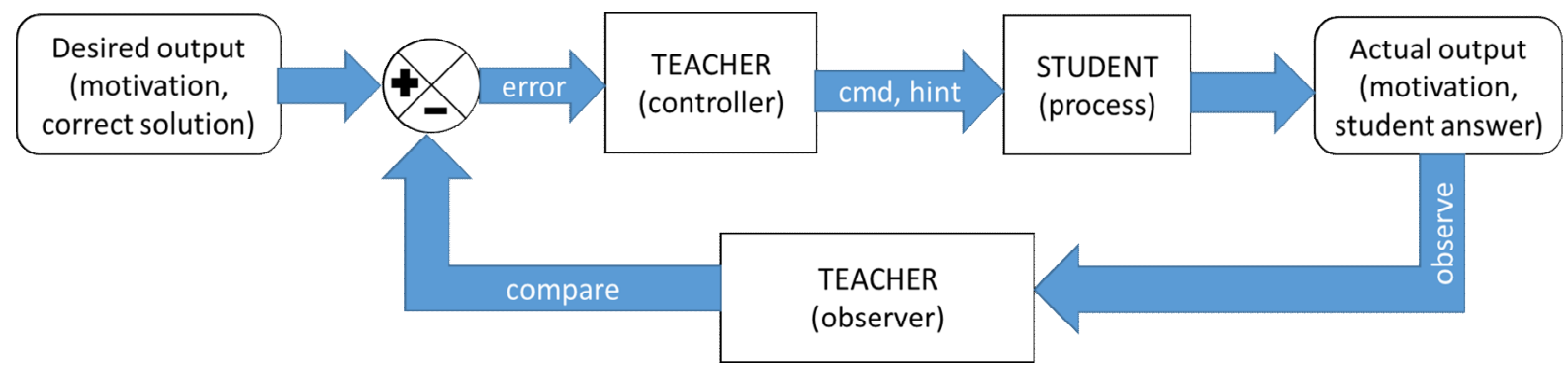

Figure 1. Closed-loop learning process with a teacher.

In a remote lab situation however, there is no teacher available (or at least not during all the remote activity), so that the learning process is an open-loop if no supplementary tools are introduced to counteract the absence of teacher. Since the tutor is replaced by the machine, substitutes are needed to play a role similar to that of the teacher (controller and observer). For that purpose, one can use sensors and computer vision to measure student mood and motivation state (sensor box). One can enrich the machine that controls and drives the learning (controller box) with new technology (use of quadcopter) or new motivating scenario (gaming). Also the output requests can be rendered more challenging (Top10) to boost the student action. Fig. 2 illustrates those ideas, showing the various functionalities added to make the loop closed again. These functionalities are presented herefater.

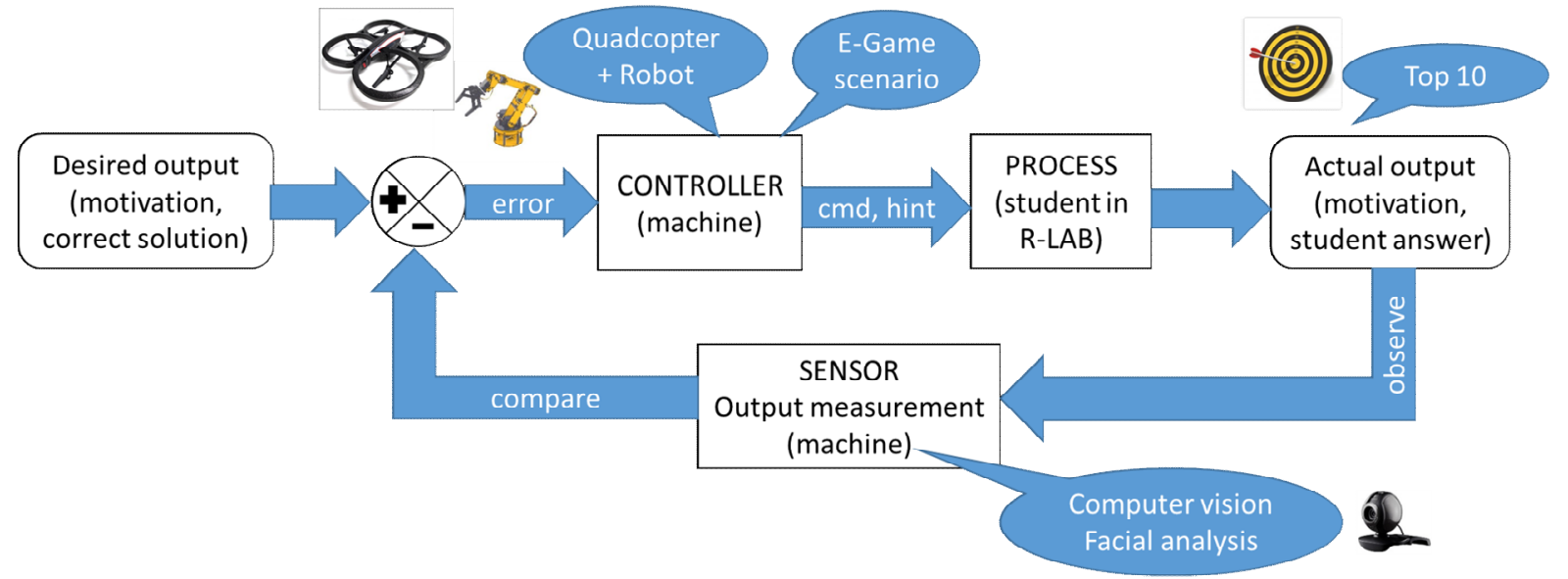

Figure 2. Without teacher, open-loop is made closed-loop again thanks to machine substitutes (in blue).

The rest of the paper is organised as follows: section 2 describes the lab platform (hardware setup) and the software interface as seen by the client (distant student), while section 3 describes the pedagocial scenario with gaming approach. Section 4 describes the visual localization system and sensors used for quadcopter 3D-pose control: a low-cost, real-time and marker-free solution is implemented. Section 5 describes the image processing and computer vision used for facial expression recognition on client side. Finally, section 6 presents the results and the evaluation of the experiments conducted with some 100 students between 2012 and 1017.

\section{REMOTE LAB TECHNOLOGY}

LaboREM is a remote laboratory in electronics developed for first year undergraduate students in engineering. The learning objective of LaboREM is to enable students to wire and test remotely 
electronic circuits, make measurements and characterize each circuit by its time or frequency response. The electronic circuits consist of operational amplifiers, active filters and oscillators.

Its design is based on a classic client-server architecture [2]. The student calls for a lab session by simple URL addressing. A first-in first-out strategy is adopted to give access to the remote lab to one client (student) at a time. A 5 minute connection is allotted to each student for one short manipulation. If he wants to repeat, he has to queue again. The remote lab application is developed using $\mathrm{NI}-$ LabVIEW software and the easy-to-use RFP protocol to pilot the remote devices. The hardware setup is shown in Fig. 3. It includes: (i) a robotic arm that mimics the student's hand for placing electronic components equipped with magnets on an electronic breadboard, (ii) measurement instruments and data acquisition system (DAQ), (iii) a webcam with zoom control that mimics the student's eye in order that the student doesn't feel so far away from what is actually happening in the lab, (iv) a quadcopter (AR quadcopter 2.0) with the role of flying in the lab for exploring the environment and inspecting electrical instruments in order to increase student immersion and motivation.

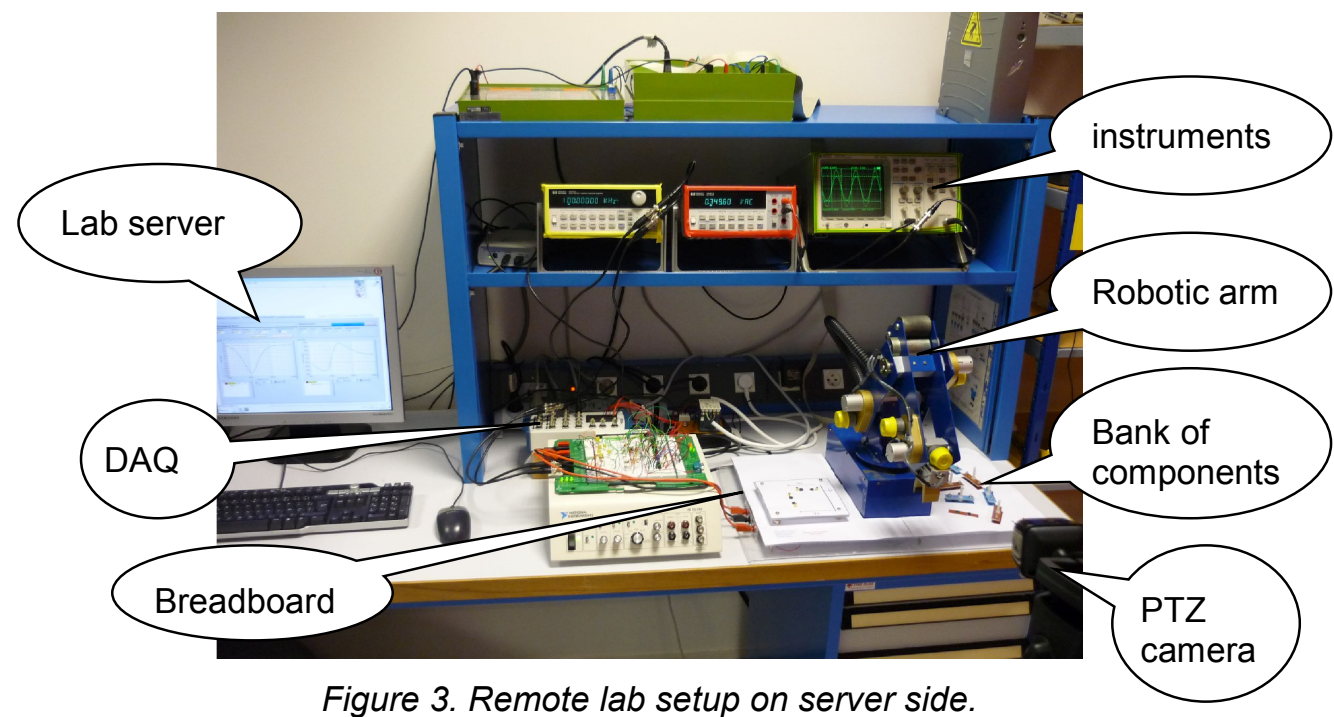

The software platform relies on the use of an LMS (Learning Management System). It gives access to the pedagogical sequence via a rich interface and collaborative tools. The learning sequence is made of various steps of activity: reading of documents, video-tutorials, quizzes, simulator, use of forum, and last but not least, access to the remote lab activity. A sample snapshot of the interface seen by the student while accessing the lab is shown in Fig. 4.

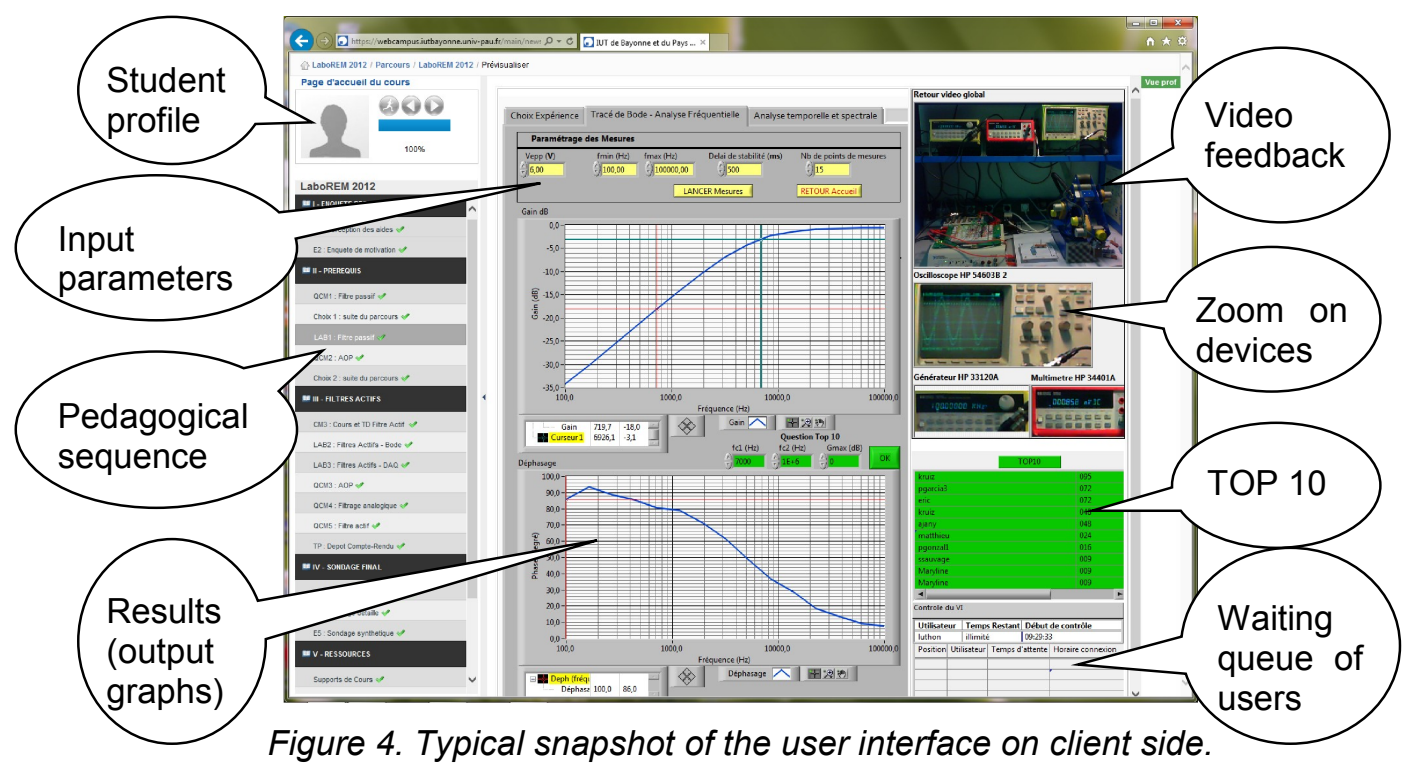

On the left column, he sees the steps of the learning sequence (with the current step in grey). On the right side, he gets a visual feedback of the lab, the current list of users that are connected or waiting to get control over the system, plus the current Top 10 scores, that evolve in real-time depending on the 
right or wrong answers to typical measures (e.g. cut-off frequency or gain of a filter under study). In the middle area, he can choose the parameters, press a bouton to run the experiment in real-time and get the results as graphical curves of gain and phase of the filter under study.

\section{GAME-LIKE PEDAGOGICAL SCENARIO}

The pedagogical scenario for the lab activity is based on a game-like approach: it includes a treasure hunt and a Top10 of the best measurements. Technically, the game scenario is built on four basic concepts like in e-games: time spent, score or mark obtained for an activity, level (beginner, medium, advanced) and number of lives or repetitions allowed. This is illustrated in Fig. 5. More details may be found in [4].

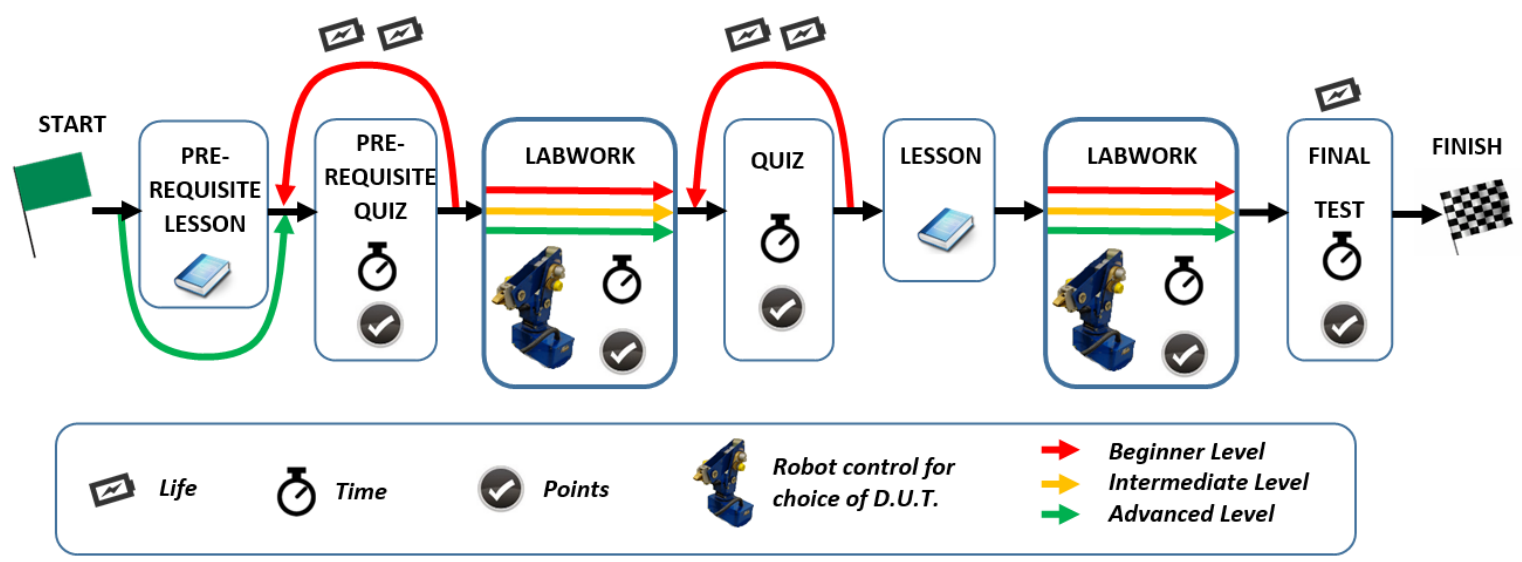

Figure 5. Map of the activity as a track-game (simplified version).

Conceptually, games are characterized by 6 key-dimensions [5]: fantasy, clear rules and goals, sensory stimuli, challenge, mystery and control. They are taken into account as follows: mystery (unknown filter to uncover), sensory stimuli (visual feedback and robotic motion), challenge (Top 10), control (choice of DUT, quadcopter remote driving), rules (limited time and number of trials), clear goals (learning objectives with three difficulty levels to choose from), fantasy (freedom to conceive one's own circuit).

The Top10 activity consists in asking to the student to give the measured value of typical parameters of the circuits (e.g. cut-off frequency). Depending on the precision of the answer (5\%,20\%), he gets more or less points that cumulate over time and make it eventually enter the "Hall of Fame" list after few or many attempts. This is illustrated in Fig. 6.

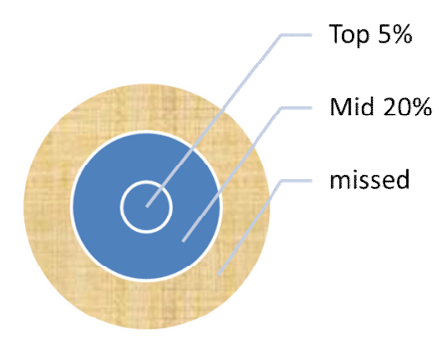

Figure 6. Best measurements participating to TOP10.

The LMS stores all the traces of the student activity (time spent, scores obtained in formative and sommative tests, level achieved, number of attempts etc.). This enables to track the student motivation and cognitive status, in order to propose him alternatives for choosing the best way to continue his travel towards the end of the lab activity. A virtual tutor (like a talking avatar appearing from time to time on the screen and giving oral advices to the student) is under investigation for implementing a smart technical solution.

This also permits to get valuable statistics to infer students profiles and make evolve the lab platform according to their needs. This will be discussed in section 6 . 


\section{QUADCOPTER CONTROL TO INCREASE STUDENT MOTIVATION}

In order to increase student motivation in remote labs, we propose to use a mini-quadcopter controlled remotely by the student. The goal is to allow the student to move in the lab, inspecting electrical instruments by sending high level commands to the mini-drone equipped with a camera. The high level commands are: (i) inspect the oscilloscope, (ii) inspect the multimeter, (iii) inspect the power supply, (iv) return to the base. In order to perform these high level commands, two main systems are necessary: (i) a localization system that localizes the mini-quadcopter in $3 \mathrm{D}$ space, (ii) a control system that sends appropriate commands to the mini-drone in order to allow it to reach a target position in 3D space. In the following subsections we present a global overview of the 2 systems and present some qualitative results.

\subsection{Visual localization sytem: A low-cost, real-time and marker-free solution}

To be able to command the mini-quadcopter to fly to a certain position in 3D space, its position in 3D space must be tracked in real-time and given to a control system. Without this, robust feedback control is not possible and the mini-quadcopter cannot reach the desired position with the desired precision and robustness. To track the 3D pose of a moving object equipped with a camera, many approaches exist. They vary in terms of complexity, real-time performance, and the need for artificial markers in the scene or additional cameras installed in the room. For example in outdoor environment, GPS is usually used to localize the drone in 3D space. For indoor environment where GPS fails to give accurate position estimate, 3D localization based on applying computer vision algorithms on the camera videostream or using other sensors (ultrasound, laser...) is usually adopted. In this work we use computer vision algorithms applied on the videostream from the camera of the quadcopter as a main sensor for localization, fusing it with inertial measurements from the sensors on-board the quadcopter in a Kalman filter framework to deal with delays and temporary loss of video signal. The system is built on the work of [6] where the authors present a SLAM system (Simultaneous Localization And Mapping) on a monocular camera that fuses the estimate from a visual algorithm with the readings from inertial sensors to localize the drone in $3 \mathrm{D}$ space with respect to a certain referential system. However this system fails when the mini-quadcopter has to closely inspect an instrument. In order to solve this problem, we use other computer vision algorithms that localize the mini-quadcopter with respect to a referential system attached to the instrument when the mini-quadcopter is close to the instrument. The idea is to use the information that the object of interest is planar and with known dimensions, to analyze its projection in the camera videostream, to estimate the pose of the miniquadcopter. In a normal configuration we use the system presented in [6]. This way, the pose of the mini-quadcopter can be tracked always and given to a control system in order to send appropriate commands.

\subsection{Feedback control system for mini-quadcopter control}

The 3D pose estimated from the computer vision algorithms plays the role of a sensor in a closed-loop feedback control system. This pose is compared to a reference pose and the error pose is given to a traditional PID controller that will calculate the commands based on this error. 4 closed-loops exist to control 4 degrees of freedom of the mini-quadcopter: (i) translation following $x$ axis (tx), (ii) translation following $y$ axis (ty), (iii) translation following $z$ axis (tz) and (iv) orientation of the drone in the horizontal plane (yaw angle). The system is presented in Fig. 7 . We evaluated qualitatively the proposed system. The system is able to control the drone for inspection, navigation and landing purposes. In Fig. 8, the inspection of an electrical instrument is illustrated. In Fig. 9, the landing manoeuvre on a planar template is illustrated. 


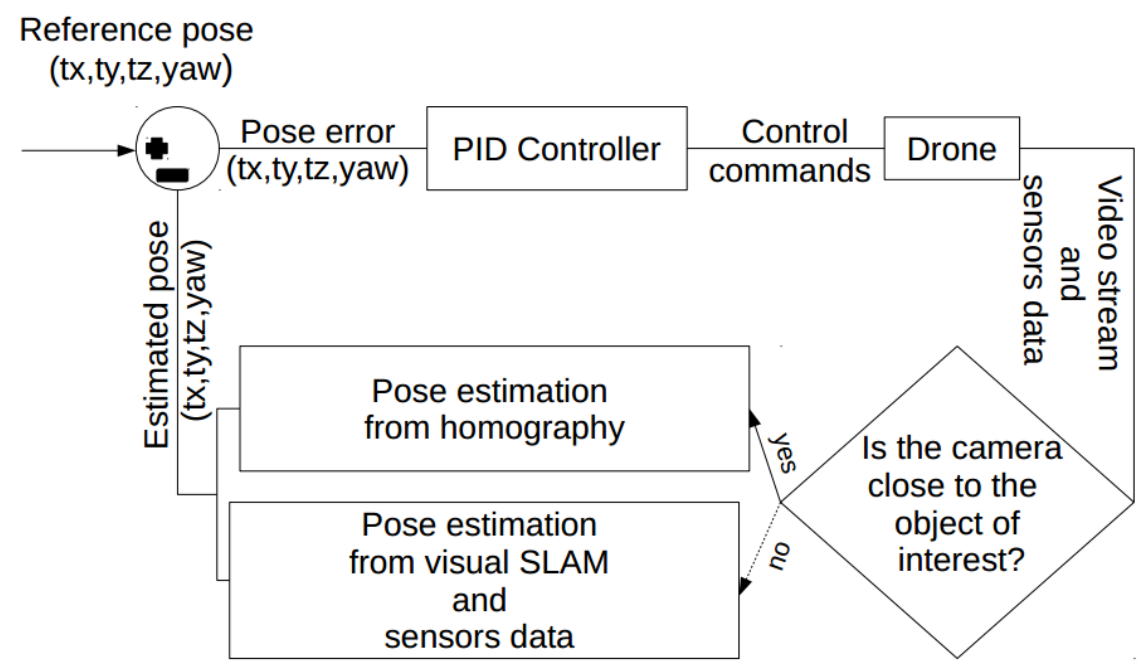

Figure 7. Feedback control system for mini-quadcopter control.
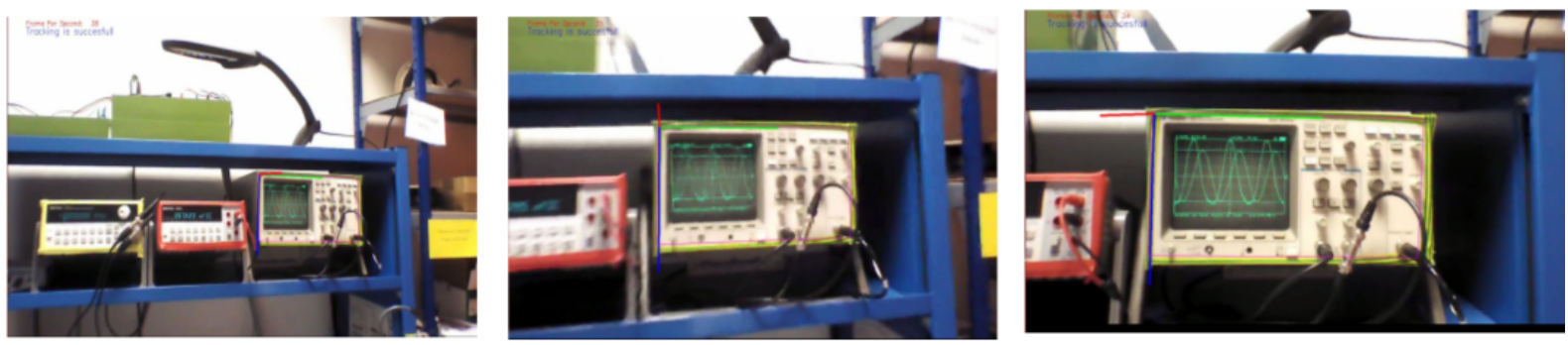

Figure 8. Localization based on an electrical instrument (oscilloscope): From left to right, images taken from the mini-quadcopter video stream that show how the system can control the mini-quadcopter to obtain a front view of the instrument.
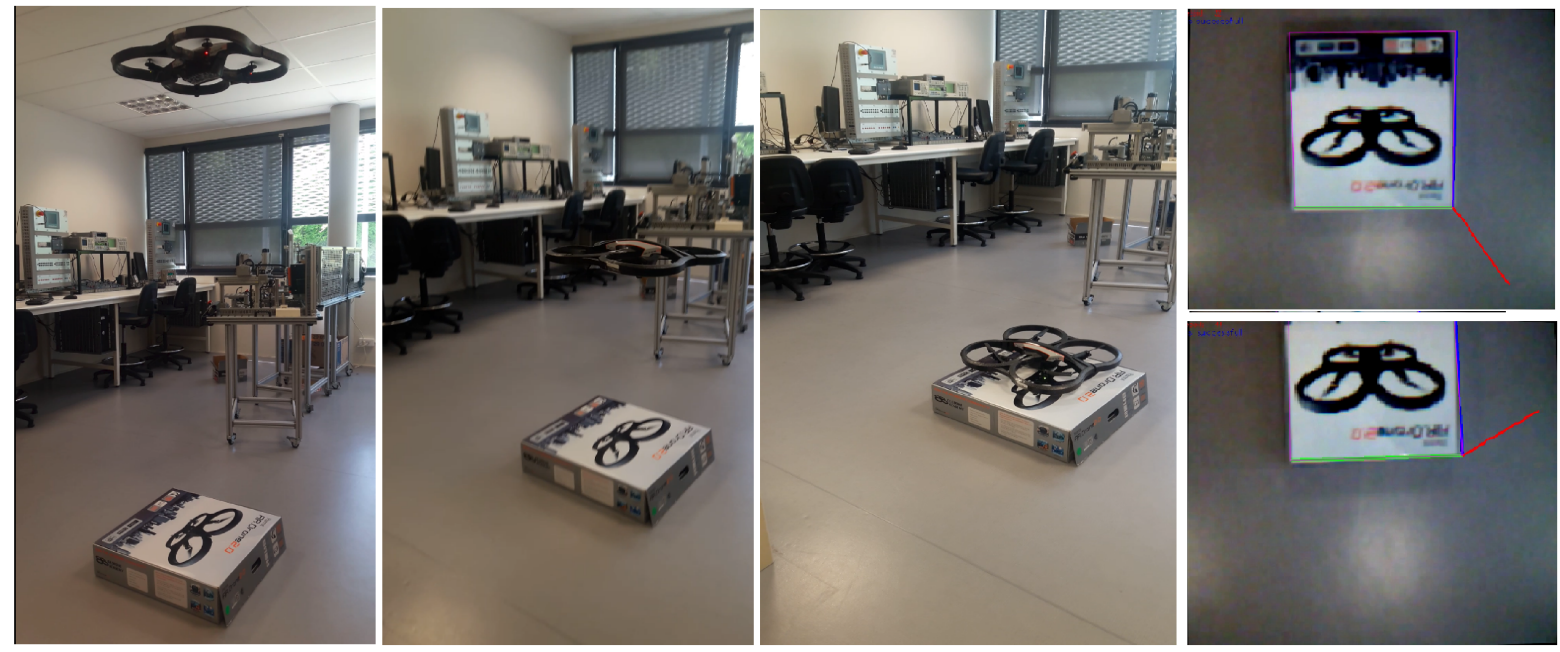

Figure 9. Visual servoing for homing. On the left: the mini-quadcopter hovering above the landing template and performing a controlled landing. On the right: the view seen from the bottom camera of the mini-quadcopter that shows robustness to clutter (when the landing object is partially out of the field of view). 


\section{IMAGE PROCESSING FOR FACIAL EXPRESSION RECOGNITION ON CLIENT SIDE}

In a remote lab, the student has to complete the labwork without the presence of a teacher. This can cause problems especially if the student faces difficulties during the lab. In the later case, the student will be demotivated, bored and unable to finish his work. To overcome this problem in remote laboratories, we propose to apply image processing algorithms on the video stream of the student's web-camera to analyse his facial expressions and head pose [7]. By detecting certain signs, a global estimate of his state can be acquired, which can help the tutoring system to make appropriate interventions to help the student in case of need. In the following section, first we present the 3D model used in our system, then we present in a general way our approach.

\subsection{D Face Model Candide}

CANDIDE is a parameterized 3D face model specifically developed for model-based coding of human faces. Its low number of polygons (approximately 100) allows fast reconstruction with moderate computing power. CANDIDE is controlled by 3 sets of parameters: global, shape and animation parameters. The global parameters correspond to the pose of the face with respect to the camera. There exist 6 global parameters: 3 Euler angles of the rotation and 3 components for the translation (tx, ty, tz). The shape parameters adjust facial features position in order to fit to different kinds of faces (eye width, distance between the eyes, face height etc). The animation parameters adjust facial features positions in order to display facial expressions and animations (smile, lowering of eyebrows). The 3D generic model is given by the 3D coordinates of its vertices $\mathrm{Pi} i=1 \ldots . \mathrm{n}$, where $\mathrm{n}$ is the number of vertices. This way, the shape, up to a global scale, can be fully described by a $3 n$-vector $g$, the concatenation of the $3 \mathrm{D}$ coordinates of all vertices:

$g=\mathrm{G}+$ STs + Ата

where $G$ is the standard shape of the model, the columns of $S$ and $A$ are the shape and animation units, TS and та are the shape and animation control vectors, respectively. The vector ts controls the static shape of the 3D model. By changing the values in Ts, the static shape of the face changes (increase width, height, position of eyes, distance between the eyes, eyes width). By changing the values in та, animation can be done (smile, eye closure, eyebrows movements). Thus in our application, values of Ts corresponding to neutral expression of a student must be determined beforehand either manually or in an automatic way taking advantage of the database of the remote lab that contains images of all the students. Fig. 10 shows the effect of 2 animation parameters on the 3D model.
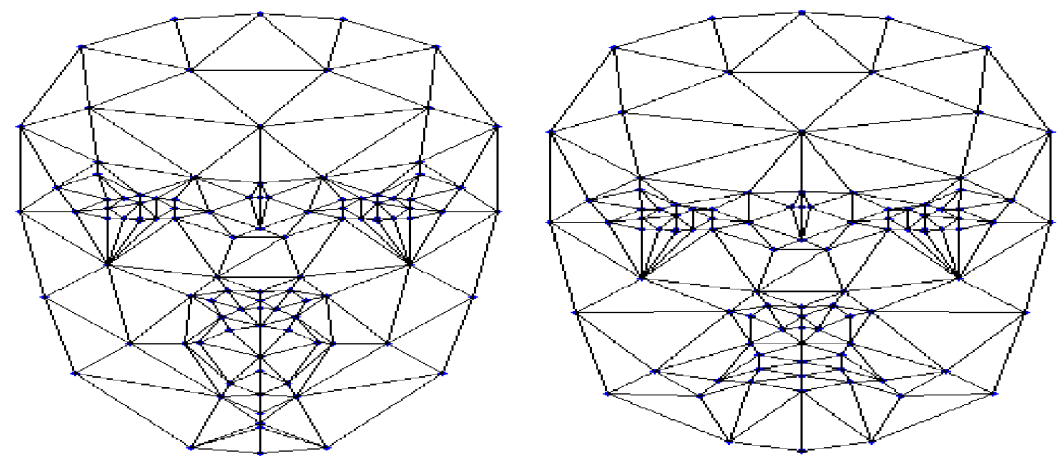

Figure 10. Candide shape with animation parameters in action.

\subsection{D Facial expression analysis and head pose estimation}

Facial expression analysis and head pose estimation from a monocular camera is a challenging subject. Many factors make this task challenging: the identity of the person, the use of a monocular camera, and the head pose. Facial appearance vary greatly with head pose. A robust algorithm for analyzing facial expressions must be able to do that for a large head pose range. In [8], the authors present a framework that is able to analyze facial expression by analyzing the displacement of facial landmarks. However this framework was restricted to a small range of head poses. One way to make facial expression analysis from monocular camera robust, is to incorporate 3D information. The 3D information is used to model the shape of human face and how it appears in the image from different 
head poses. In our work we use a 3D deformable Candide model that can be adapted to every human in order to take his face shape. The idea is to put in correspondence 3D points of the face model and $2 \mathrm{D}$ points detected in the image. From this correspondence and by estimating the projection matrix that maps the $3 D$ points to the $2 D$ points based on an orthographic projection, the pose of the human head with respect to the webcam can be estimated. After this step, the neutral face model is projected and fitted to the image allowing for animation parameters estimation and thus an indication of the facial expression currently occurring. The 2D points are detected using the algorithm in [9]. Fig. 11 shows the framework used for facial expression analysis and head pose estimation. Fig. 12 shows the 3D mesh fitted to a student face.

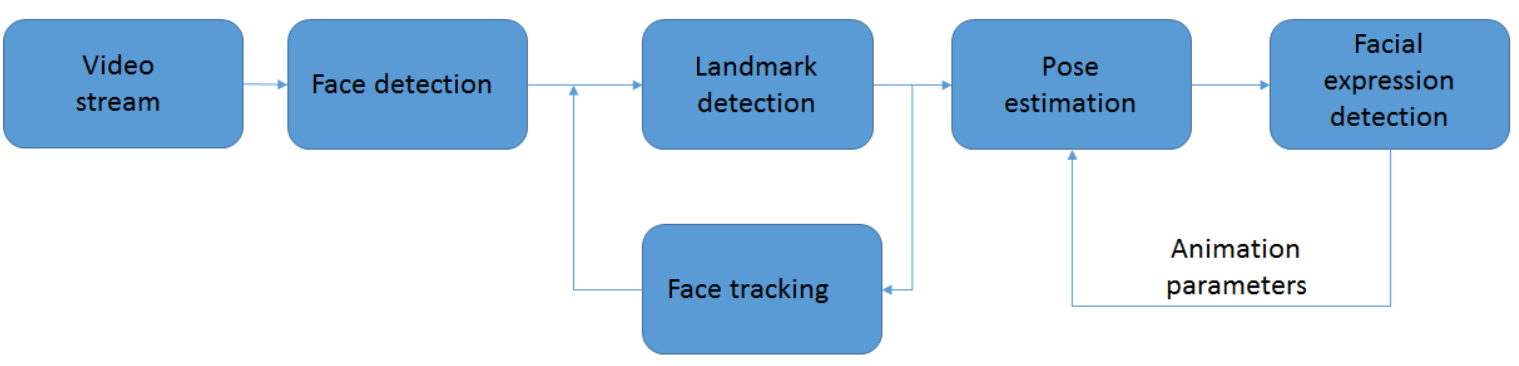

Figure 11. Facial expression and head pose estimation framework.

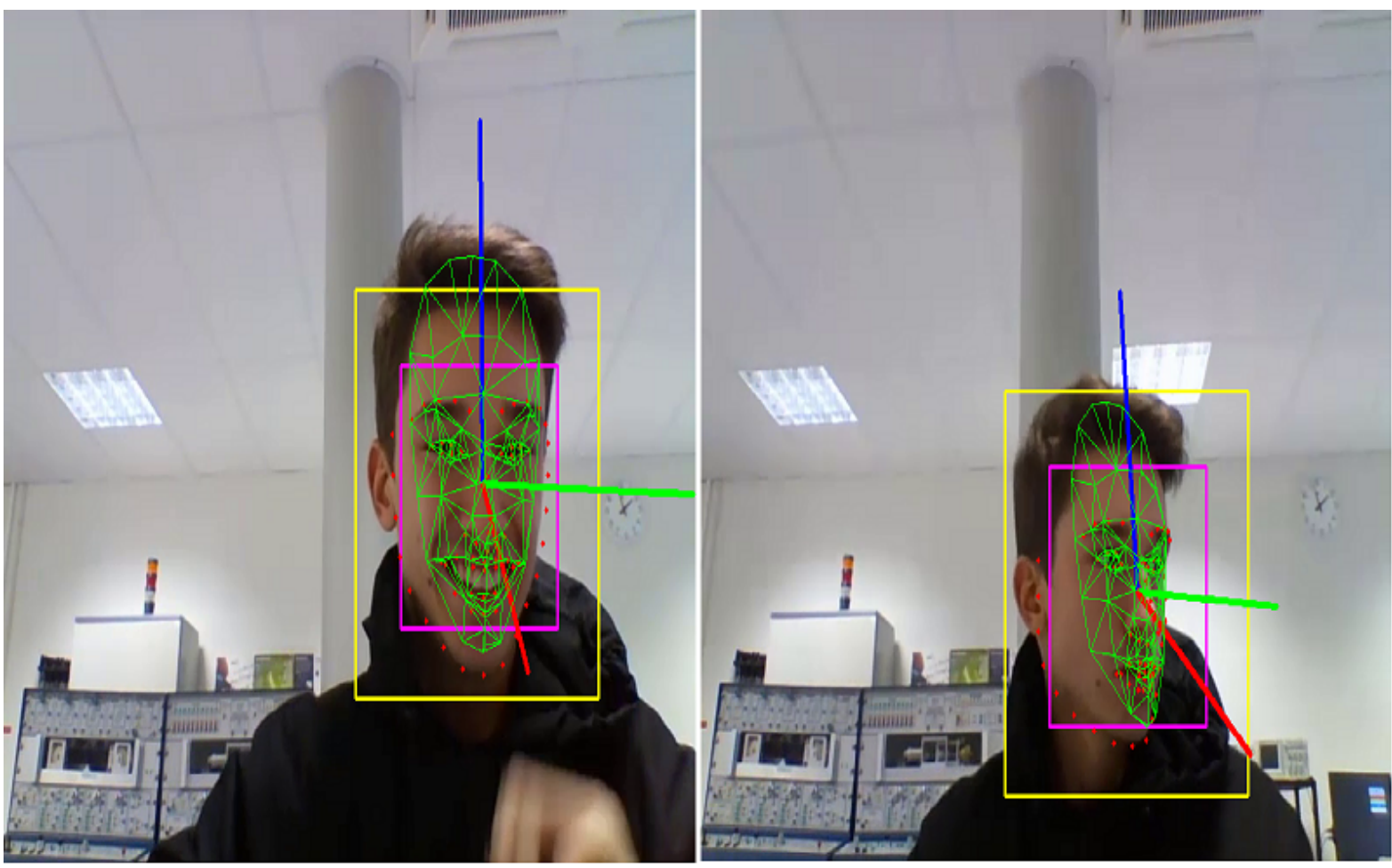

Figure 12. 3D face model fitted to a student face.

\section{RESULTS}

\subsection{Student surveys}

As part of the pedagogical sequence, there are 5 questionnaires (some of them being anonymous) to be filled-in by the students at the beginning (priori inquiry) or at the end (posterior inquiry) of the labwork. Hereafter, some of the most significant results are listed.

\subsubsection{Result 1 about platform evaluation}

There is an overall improvement of the platform quality over the years (new features being added like Top10, robot, simulator): the mean-score of the anonymous student inquiry from 2012 to 2017 is shown in Tab.1. The score is given on a 20-point scale ( 0 being the worst, and 20 being the best). 
Table 1. Qualitative evaluation of the platform by the students (on a 20-point scale).

\begin{tabular}{l|c|c|c}
\hline \hline Year (\# students) & $2012(22)$ & $2013(25)$ & 2014 \& $2017(44)$ \\
\hline Comfort of interaction & 12.3 & 13 & 13.8 \\
\hline Technical quality & 12.8 & 13.5 & 14.0 \\
\hline Game-like scenario & 10.4 & 13.4 & 13.6 \\
\hline Pedagogical efficacy & 10.3 & 12.6 & 13.2 \\
\hline Remote control of instruments & 11.7 & 14.1 & 14.5 \\
\hline Space and time freedom & 13.1 & 12.4 & 16.4 \\
\hline Collaborative work & 13.4 & 15.2 & 13.5 \\
\hline Autonomy & 12.3 & 14.2 & 13.6 \\
\hline Preparation and prerequisites & 14 & 14.5 & 13.0 \\
\hline Overall satisfaction & 10.7 & 13.3 & 14.0 \\
\hline Mean Scoring & 12.1 & 13.6 & 14.5 \\
\hline \hline
\end{tabular}

Anonymous comments of the students are also collected. They are globally positive about this activity, but still mention the importance of hands-on labs and of collaboration between students or with a tutor. Indeed, the best solution for learning is blended learning (i.e. a mix of various modalities: hands-on, simulation, distance). Moreover, they often mention technical bugs due to newtork problems, which remain an important issue for R-LABs (bad configurations of webbrowser, plugins, fire-walls etc.)

\subsubsection{Result 2 about online help needs}

Survey about help or assistance needs is strongly coherent from 2012 to 2017 (see Tab. 2). Students are mainly proactive: they accept some help and assistance, but want to remain actor of their activity. The survey is based on 19 assertions like: "I will feel incompetent if I use online help in LaboREM" or "Using online help in LaboREM will be usefull for my learning" etc.

Table 2. Survey about the use of help (on a 5-point Likert scale: 1="Not at all" ; 5="definitely Yes")

\begin{tabular}{l|c|c|c|c}
\hline \hline Year (\# students) & $2012(22)$ & $2013(25)$ & $2014(17)$ & $2017(44)$ \\
\hline Passive, assisted: complete help needed & 2.44 & 1.93 & 2.8 & 2.23 \\
\hline Negative: stubborn refusal of help & 2.04 & 1.90 & 1.67 & 1.98 \\
\hline Autonomous: no need of help & 2.24 & 2.43 & 2.39 & 2.23 \\
\hline Proactive: ok for partial help & 3.6 & 3.80 & 3.53 & 3.94 \\
\hline \hline
\end{tabular}

\subsubsection{Result 3 about student motivation}

The a priori motivation of our students is mainly intrinsic, meaning that they are little motivated by external factors (Tab. 3). This survey is based on 14 assertions like "It is important for me that other students think I am competent" or "It is important for me to improve my own proficiency". In 2013, the Top10 and the robotic arm were added on the platform: this proved to increase the a posteriori extrinsic motivation. 
Table 3. Survey about motivation (on a 5-point Likert scale).

\begin{tabular}{l|c|c|c|c}
\hline \hline Year (\# students) & $2012(22)$ & $2013(25)$ & $2014(16)$ & $2017(40)$ \\
\hline Intrinsic a priori & $\mathbf{3 . 8 9}$ & $\mathbf{4 . 1 8}$ & $\mathbf{3 . 8 5}$ & $\mathbf{4 . 3 3}$ \\
\hline Extrinsic a priori & 1.95 & 2.42 & 1.94 & 2.27 \\
\hline Extrinsic a posteriori & 2.55 & 3.1 & 2.69 & 3.16 \\
\hline \hline
\end{tabular}

\subsection{Statistics about student achievements}

\subsubsection{Result 4 about Top10}

There is a strong positive correlation between the time spent and the Top10 score. Indeed, the Pearson coefficient is equal to $r=0.82$ (for 44 students in 2017). Whereas the correlation between time spent and global mark obtained at the activity (including also quizzes, written report etc.) is not so strong ( $r=0.67)$. This seems to indicate that the Top10 is indeed very motivating: students are willing to spend time in order to appear in the Top10 list. In the meanwhile, they learn circuit characterization.

\section{CONCLUSION}

The LaboREM prototype still needs improvements, both technical and pedagogical. Nevertheless, the results obtained until now show that adding new tools like robot, computer vision, top10 etc. tends to increase student motivatioon for learning, which is an important issue at undergraduate level.

\section{ACKNOWLEDGEMENTS}

We thank our students, and especially Lucas Citerin, for accepting to get filmed during the labwork.

\section{REFERENCES}

[1] J. Garcia-Zubia, G. R. Alves, Using remote labs in education: two little ducks in remote experimentation. University of Deusto, Bilbao, Spain, 2011, ISBN 978-84-9830-398-8.

[2] F.Luthon, B. Larroque, "LaboREM - A remote laboratory for game-like training in electronics," IEEE Transactions on Learning Technologies, vol. 8, no. 3, pp. 311-321, Jul. 2015.

[3] J.J. Gonzalez, "A closed-loop educational system for teaching electronics: replacing homework assignments with design mini-projects," $8^{\text {th }}$ Int. Conf. of Education, Research and Inovation (ICERI2015), pp. 5199-5205, Seville, Spain, 16-18 Nov. 2015.

[4] F. Luthon, B. Larroque"Real labworks in electronics: Yes! ... But remotely controlled," $8^{\text {th }}$ Int. Conf. of Education, Research and Inovation (ICERI2015), pp.8490-8500, Seville, Spain, 16-18 Nov. 2015

[5] R. Garris, R. Ahlers, J. E. Driskell, "Games, motivation, and learning: a research and practice model," Simulation \& Gaming, vol.33, no.4, pp. 441-467, Dec. 2002.

[6] Engel, Jakob, Jürgen Sturm, and Daniel Cremers, "Scale-aware navigation of a low-cost quadcopter with a monocular camera," Robotics and Autonomous Systems 62.11 (2014): 16461656.

[7] F. Khattar, F. Luthon, B. Larroque, F. Dornaika, "Using computer vision for student-centred remote lab in electronics," 8th Int. Conf. on Education and New Learning Technologies (EDULEARN16), pp. 614-623, Barcelona, Spain, 4-6 July 2016.

[8] M. Pantic, I. Patras, " Dynamics of Facial Expression: Recognition of Facial Actions and Their Temporal Segments From Face Profile Image Sequences," IEEE Trans. Systems, Man, and Cybernetics - Part B: CYBERNETICS, vol. 36, no. 2, pp.433-449, April 2006.

[9] Kazemi, Vahid, and Josephine Sullivan, "One millisecond face alignment with an ensemble of regression trees," Proc. IEEE Conference on Computer Vision and Pattern Recognition. 2014. 